\title{
Anticancer Activities of Hesperidin and Hesperetin In vivo and their Potentiality against Bladder Cancer
}

\author{
Danijela Stanisic ${ }^{1}$, Amanda F. Costa1, Wagner J. Fávaro ${ }^{2,3}$, Ljubica Tasic ${ }^{1,3}$, Amedea B. Seabra ${ }^{3,4,5}$ and Nelson Durán ${ }^{2,3,4,5 *}$ \\ ${ }^{1}$ Biological Chemistry Laboratory, Institute of Chemistry, University of Campinas, Campinas, SP, Brazil \\ ${ }^{2}$ Department of Structural and Functional Biology, Laboratory of Urogenital Carcinogenesis and Immunotherapy, University of Campinas, Campinas, SP, Brazil \\ ${ }^{3}$ NanoBioss - Institute of Chemistry, University of Campinas, Campinas, SP, Brazil \\ ${ }^{4}$ Nanomedicine Research Unit, Federal University of ABC, Santo André, Brazil \\ ${ }^{5}$ Center for Natural and Human Sciences, Federal University of ABC, Santo André, SP, Brazil
}

\begin{abstract}
Hesperidin and its aglycone hesperetin have emerged as important anticancer natural products from orange resources, while bladder cancer has been a great treat to animals and humans in very severe forms. Bladder cancer is usually treated with extremely toxic chemotherapeutics, such as doxorubicin, cisplatin, mitomycin- $\mathrm{C}$, etc., and there is as an urgent need for new chemotherapeutics for its treatment. An important strategy to minimize the toxic effects of the above cited drugs is to use co-adjuvant. Hesperidin and hesperetin have shown promising results in the suppression of various types of cancer (colon, prostate, hepatic, bladder and lung cancer), as a drug or as pro-drug and co-adjuvants. This mini-review points to the importance of flavonoids in different cancers' treatments, with special attention on the combat of bladder cancer.
\end{abstract}

Keywords: Hesperidin; Hesperetin; Bladder cancer; Oranges; Chemotherapeutics

\section{Introduction}

Hesperidin (3',5,7-trihydroxy-4'-methoxy flavanone-7-6-O- $\alpha-L-$ rhamnosyl-D-glucose) (Figure 1) is a naturally occurring flavonoid found abundantly in vegetables and fruits [1]. It is a cheap byproduct of citrus production and one of the most important bioflavonoids in sweet orange and lemon [2]. Hesperidin exhibits many beneficial effects such as anti-allergic, anti-oxidant and anti-inflammatory actions [3]. Anticarcinogenic effects in tongue, esophagus, colon and urinary bladder in rat models of carcinogenesis were reported [4]

Hesperetin (3',5,7-trihydroxy-4'-methoxy flavanone) is also a natural flavonoid, an aglycone of hesperidin (Figure 1), which has been reported to exert similar biological activities when compared to hesperidin and interesting therapeutic value in neuropathological conditions [5].

\section{In vivo Anticancer Activities of Hesperidin and Hesperetin}

Phytochemicals derived from hesperidin have important chemoprotective roles in carcinogenesis. There are many types of

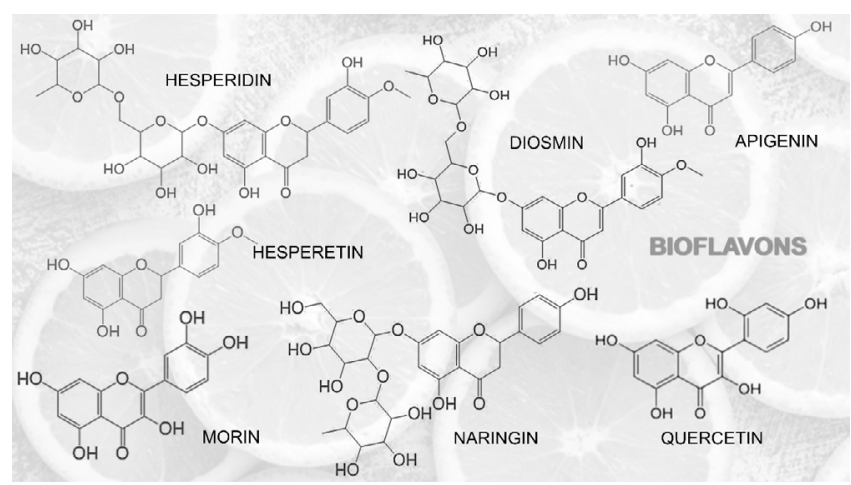

Figure 1: Bioflavones from oranges are potentially great adjuvants in anticance treatment and, in bladder cancer therapy minimize toxic chemotherapeutics effects. Illustrated are the structures of already tested bioflavones in vivo. We emphasize the positive effects of hesperetin and hesperidin. cancers in which it is known that hesperidin can act effectively, such as colon cancer (adenoma and adenocarcinoma), breast cancer, melanoma, prostate cancer, hepatocellular carcinoma, and many others, as well as in metastasis reduction. It is also known that in lung, liver, breast, stomach, and colon cancer, hesperidin probably acts through the promotion of cancer cells apoptosis via multiple mechanisms [6].

Arafa et al. reported the effect of hesperidin on benzo[a]pyrene $(\mathrm{BaP})$ that induced testicular toxicity in rats. The authors showed the BaP (orally administrated) diminished the testicular activities of superoxide dismutase (SOD), glutathione S-transferase (GST), and testicular glutathione (GSH) and increased malondialdehyde (MDA) contents. Hesperidin orally applied as pretreatment ameliorated all the biological and histological changes induced by $\mathrm{BaP}$. As well, hesperidin had a protection effect $v s$. lung cancer induced by BaP in mice [7].

Saiprasad et al. reported the chemopreventive potential of hesperidin against an inducer of colon carcinogenesis (azoxymethane - AOM) in mice model. Hesperidin orally applied in mice inhibited the nuclear factor-kappa B (NF-kB) dependent inflammatory responses including inducible nitric oxide synthase (iNOS) and cyclooxygenase-2 (COX-2) activation [8]. More recent studies related to elucidation of molecular mechanism indicated that hesperidin induced apoptosis and cell cycle arrest in AOM that produce mouse colon carcinogenesis via inhibiting Phosphoinositide 3-kinase/Protein kinase B (P13K/ Akt) pathway and inhibiting Arurora-A and Akt mediated GSK-3 $\beta / \beta$ -

*Corresponding author: Nelson Durán, Center for Natural and Human Sciences, Federal University of ABC, Santo André, SP, Brazil, Tel: 55713319350; E-mail: duran@iqm.unicamp.br

Received: September 08, 2018; Accepted: October 03, 2018; Published: October 08, 2018

Citation: Stanisic D, Costa AF, Fávaro WJ, Tasic L, Seabra AB, et al. (2018) Anticancer Activities of Hesperidin and Hesperetin In vivo and their Potentiality against Bladder Cancer. J Nanomed Nanotechnol 9: 515. doi: 10.4172/2157 7439.1000515

Copyright: $\odot 2018$ Stanisic D, et al. This is an open-access article distributed under the terms of the Creative Commons Attribution License, which permits unrestricted use, distribution, and reproduction in any medium, provided the original author and source are credited. 
catenin cascade. $\beta$-catenin is an oncoprotein that plays a pivotal role in AOM mediated colon tumor development in mice [9]. Hesperidin altered AOM mediated anti-apoptotic process by inflecting the Bax: $\mathrm{Bcl} 2$ ratio, as well as, inducing an increase in cytochrome-c level and activation of caspases 3 and 9 . Beside these, hesperidin increased the level of p53 and p21 expressions, thus, suppressing tumor. Moreover, hesperidin blocked the signaling $\mathrm{f}$ cascade phosphoinositide-3-kinase (PI3K)/Akt by up-regulating the expression of PTEN- Phosphatase and tensin homolog (tumor suppressor protein) and by inhibition of AuroraA (upstream regulator of PI3K/Akt pathway). Suppression of P13/Akt pathway induces apoptosis and inhibits tumor progression. Furthermore, the administration of hesperidin stimulated autophagic markers, such as, Beclin-1 and LC3-II by mTOR protein (mammalian target of rapamycin) inhibition, which are important players in autophagy mediated cell death. Beside these, hesperidin prevents the build-up of $\beta$-cateninin cytoplasm by activating of glycogen synthase kinase-3 beta (GSK-3). Many authors concluded that hesperidin exerts its major role in AOM mediated colon carcinogenicity by occluding the Aurora-A mediated PI3K/Akt/GSK3 and mTOR signaling pathways by promoting apoptosis and autophagy mediated cell death in mice $[10,11]$.

Still, various signaling pathways as MAPKs (Mitogen-activated protein kinases) and NF-kB are hyper active in inflammatory cells, which exert a pivotal role in the expression of COX-2, iNOS, TNF, and IL-6 [12,13]. Many studies suggest that hesperidin acts as an antiinflammatory agent against several inflammatory mediated diseases, such as cancer. Hesperidin exerts the anti-inflammatory activity by targeting many inflammatory components (IL-6, TNF, COX-2, iNOS, etc), that are involved in tumor progression [14]. Hesperidin treatment $(50 \mathrm{mg} / \mathrm{kg})$ reversed the levels of TNF- and IL-1 in the carcinogen 2,3,7,8-tetrachlorodibenzo- $p$-dioxin (TCDD) treated rats [15]. In a similar response, administration of citrus juices (high content of hesperidin) in NKK (4-(methylnitrosamino)-1-(3-pyridyl)-1butanone) induced lung and colon carcinogenesis in mice and rats. The citrus juice also produced a down-regulation of mRNA expression of various cytokines, such as, TNF, IL-1, IL-6 and inflammatory enzymes (iNOS and COX-2) and mRNA up-regulation of Nrf2 (Nuclear factor-2), quinine reductase and glutathione S-transferase, which neatly confirmed the hesperidin anti-inflammatory mechanism against NKK induced cancer [16]. Moreover, hesperidin $(30 \mu \mathrm{M})$ decreased the level of nitrogen dioxide $\left(\mathrm{NO}_{2}\right)$ and repressed the expression of iNOS protein when added in LPS-induced RAW264.7 inflammation model as discussed by Sakata et al. [17]. Hesperidin prevented azoxymethane (AOM)-induced rat colon carcinogenesis [4]. In this case, rats were fed a diet containing hesperidin (1000 ppm or $1.6 \mathrm{mM})$, and the observed cancer interruption could be related to the inhibition of the increased cell proliferation produced by the carcinogens in the affected mucous membranes.

Hesperetin (3',5,7-trihydroxy-4'-methoxy flavanone) is an aglycone part of hesperidin. Oral administration of hesperetin diminished the number of lung metastases in C57BL6/N mice inoculated with B16F10 cells, and enhanced survival time after tumor cell inoculation [18]. Hesperetin inhibits the proliferation and induces the apoptosis of hepatocellular carcinoma through triggering the activation of the mitochondrial pathway by rising levels of intracellular reactive oxygen species (ROS), $\mathrm{Ca}^{2+}$ and ATP on male Balb/c-nu/nu nude mice. This report identified the utility of hesperetin, as a safe and nontoxic antitumor agent, in the colon cancer treatment [19]. Furthermore, hesperetin suppressed efficiently the growth of xenograft tumors in mice model of gastric cancer [20]. Hesperetin exerted an inhibitory effect on cell nuclear antigen proliferation in ACF in the induction of colon cancer model rats by 1,2-dimethylhydrazine (DMH) [21]. Also, it inhibited growth of aromatase-expressing MCF-7 tumor in ovariectomized athymic mice by decreasing cyclin D1, CDK4, and Bcl$\mathrm{x}(\mathrm{L})$, when upregulating the level of $\mathrm{p} 57^{\mathrm{Kip} 2}$ [22].

Hesperetin treatment of C6 gliomas caused a marked decrease in the tumor permeability and edema and increased expression of tight junction-associated proteins. Hesperetin treatment also downregulated the HIF-1a/VEGF/VEGFR2 pathway and it was concluded that hesperetin possesses anti-tumor properties in implanted C6 glioma cells in rats [23].

The combination of hesperetin and nanomaterials represents a new strategy to treat cancer cells. In this sense, hesperetin conjugated with gold nanoparticles (Au-mPEG(5000)-S-HP NPs) acting on diethylnitrosamine (DEN)-induced hepatocarcinogenesis in male Wistar albino rats was studied. DEN-administered animals exhibited increased mast cell counts, transcription factor nuclear factor- $\kappa \mathrm{B}$, tumor necrosis factor alpha, glycoconjugates, argyrophilic nucleolar organizing regions and proliferating cell nuclear antigen. While $\mathrm{Au}-$ mPEG(5000)-S-HP NPs supplementation efficiently suppressed all the observed abnormalities. These results indicates that the AumPEG(5000)-S-HP NPs exhibited significant potential anticancer activity by suppressing cell inflammation and proliferation in DENinduced hepatocellular carcinogenesis [24]. Similarly, hesperidin at concentration of $20 \mathrm{mg} / \mathrm{kg}$ BW, inhibited cell proliferation markers, COX-2 mRNA expression, angiogenic growth factors, enhanced apoptosis, and reduced aberrant crypt foci in DMH-induced colon carcinogenesis in rats [25]. Western blot analysis indicated that cyclin D1, CDK4, and Bcl-XL were reduced in the tumors in breast cancer of hesperetin-treated mice, the results suggested that flavanone reduced plasma estrogen [26]. Hesperetin was also reported to modulate xenobiotic-metabolizing enzymes on $\mathrm{DMH}$-induced colon carcinogenesis [27].

Rossi et al. provided case-control study and obtained important results in subgroups of population, in special for flavanones, and these data should be taken with caution as statistical results might not be sufficient to detect meaningful associations. Previously, no study investigated the relation between flavanones and endometrial cancer risk, in respect of their estrogenic and anti-estrogenic activity [28].

This section highlighted that hesperidin and hesperetin have potent effects against different types of cancers. As our interest is on bladder cancer, the next sections present and discuss the recent progress on the uses chemotherapeutic regimens to combat bladder cancer and the potent uses of hesperidin and hesperetin against bladder cancer.

\section{Bladder Cancer}

The standard of care for bladder cancer has presented just a few changes over the last decades and treatment options remain limited. Some treatments, such as cystectomy, imply drastic lifestyle changes that diminish the patients' quality of life while falling far short of achieving cure. The standard conservative treatments surgical resection of urinary bladder followed by intravesical Bacillus Calmette-Guerin (BCG) immunotherapy prevent the progression of high-grade nonmuscle invasive bladder cancer (NMIBC) to advanced disease. But, often the efficacy is weakened by the emergence of refractory or relapsing disease, and toxicity causes the discontinuation of the treatment. Radical or partial cystectomy is the option for non-responsive patients to the current bladder-sparing therapies. 
Citation: Stanisic D, Costa AF, Fávaro WJ, Tasic L, Seabra AB, et al. (2018) Anticancer Activities of Hesperidin and Hesperetin In vivo and their Potentiality against Bladder Cancer. J Nanomed Nanotechnol 9: 515. doi: 10.4172/2157-7439.1000515

Patients who refuse or are not eligible for bladder removal face a dismal prognosis, due to the increased risk of progression to advanced disease. Despite conservative treatments, a large number of NMIBC patients at the time of diagnosis will develop invasive or metastatic disease. Around half of patients with specific advanced or metastatic bladder cancer do not respond satisfactorily to first-line platinumbased chemotherapies, that is, methotrexate, vinblastine, doxorubicin (adriamycin) and cisplatin (MVAC), and gemcitabine and cisplatin (GC) (Figure 2)

Due to cisplatin's high toxicity, many patients will receive doses lower than those typically recommended or even no treatment. The alternative would be a carboplatin-based chemotherapy, as secondline treatment (gemcitabine plus carboplatin or gemcitabine plus paclitaxel), which provides a median survival of 9-10 months [29]. Despite the first-line platinum-based therapy, options are quite few for those patients whose malignant lesions progress. Only $10 \%$ to $15 \%$ of them respond to second-line single-agent chemotherapy [29].

It is known from the literature that many of the anticancer drugs are very toxic and the role of the flavonoids might be important to ameliorate their toxicity. For example, doxorubicin (DOX) is one of the effective cytotoxic drugs applied in cancer therapy, but, cumulative cardiotoxicity and nephrotoxicity confines its clinical applications. Systemic application of drugs given by intravesical therapy mostly reach the inner part of the bladder, with little-to-no effect on other organs, such as the liver, heart, lung, spleen, kidneys, ureters, and urethra. It is known that intravesical chemotherapy can maximize the exposure of tumor to any therapeutic agents while limit the drug systemic toxicity due to the lumen structure of the bladder. For example, nanoparticles prepared with cationic 1,2-dioleoyl-3-trimethylammonium propane/ methoxypoly (ethylene glycol) (DPP) to give up doxorubicin (DOX) for intravesical administration of bladder cancer were studied. The DPP micelles were able to extend the residence of DOX in the bladder, increase the penetration of DOX into the bladder wall, and enhance cellular uptake of DOX. DOX encapsulation by DPP micelles extensively improved the in vivo anticancer effect of DOX against orthotopic bladder cancer [30].

Cisplatin (CIS) is the main player in chemotherapy of muscle invasive urinary bladder cancer (MIBC). CIS as monotherapy in MIBC is considerate as less efficient than associated therapy.

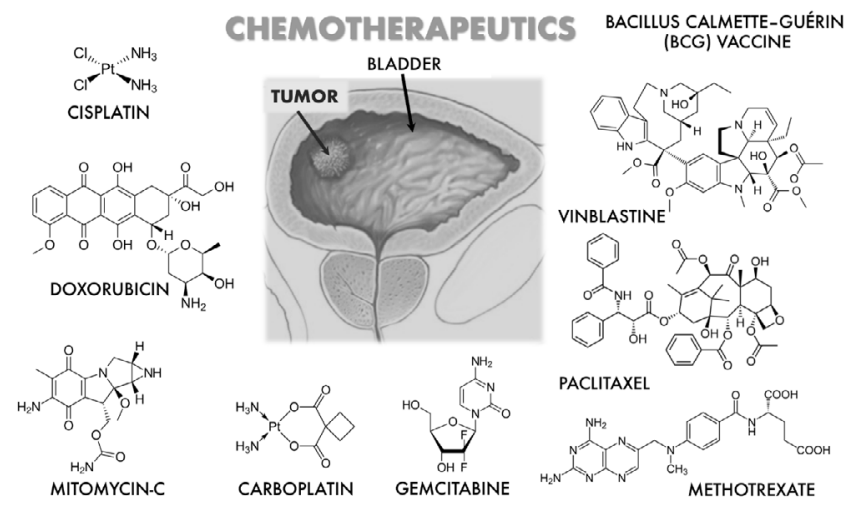

Figure 2: Bladder cancer can show different stagings and its common anticancer treatment includes: surgery, radiotherapy, immunotherapy and chemotherapy. Chemotherapeutics are divided into the first and secondgeneration substances, which often present many toxic effects. Structures of the most used anticancer agents are shown and for further information see text.
CIS is also considered a Gold standard following the data of the randomized prospective trials and too in a major meta-analysis of all randomized controlled trials addressing the concept (RCTs). Basic immunomodulatory effects of CIS are:

- Raised the major histocompatibility complex (MHC) class I expression, which is important for tumor cell recognition and deletion by CD8+ cytotoxic T cells, and enhanced expression would thus therefore promote anti-tumor immune responses.

- Enrollment and proliferation of effectors' cells is of essential importance for an efficient immune response to happen. Experimental investigations indicate CIS as a facilitator and promotor in the homing process leadings substantially increased amounts of activated cytotoxic T cells (CTLs), both systematically and locally in the tumor.

- Increase of tumor-lytic activity of cytotoxic effectors. A previous treatment of CTLs with CIS has been shown to enhance CTL-mediated killing of tumor cells in cells lines, such as lymphoblastoid cell lines and in other cells.

- Down regulation of immunosuppressive agents in the microenvironment. CIS has been demonstrated to down regulate either myeloid-derived suppressor cells (MDSC) and $\mathrm{T}$ regulatory cells (Treg) in different studies, with suggested secondary effects on immunocompetent cells being unobstructed in their respective activity. Data indicate that CIS has a function of revealing and exhibiting subdominant epitopes of tumor antigens. Thereby, increasing the possibility of the adaptive immune system (CTLs) to recognize else hidden tumor targets [31].

Unfortunately, CIS exerts many side effects (occurring in greater than 30\%) for patients: nausea and vomiting, kidney toxicity, ototoxicity, low blood counts and blood test abnormalities. The side effects that occur in 10\%-29\% for patients receiving CIS are peripheral neuropathy, taste changes, loss of appetite, hematological problems and hair loss (http://chemocare.com/chemotherapy/ drug-info/ cisplatin.aspx). CIS treatment also can lead to significant reduction on the enzymatic activity of antioxidant enzymes like SOD, glutathione peroxidase $(\mathrm{GPx})$, catalase (CAT), glutathione S-transferases (GST) and glutathione reductase (GR)

\section{Potential Effects of Hesperidin and Hesperetin and other Flavonoids on Cancer}

This section highlights the beneficial effects of hesperidin and hesperetin associated with traditional chemotherapeutic drugs in different cancers.

Abdel-Raheem and Abdel-Ghany in 2009 studied the protective effect of hesperidin against cardiotoxicity in rats by DOX treatment [32]. Imbalance in nitric oxide (NO) generation and increased ROS have been implicated in the cardiotoxicity of doxorubicin. These results demonstrated significant decrease in glutathione (GSH) levels, superoxide dismutase (SOD) activity, and enhance in thiobarbituric acid reactive substances (TBARS) levels, showing that doxorubicininduced cardiotoxicity was mediated through reactive oxygen species (ROS) generation. These findings disclose pretreatment with hesperidin reversed negative effects induced by DOX [33]

Hesperidin diminished CIS-induced functional and histopathological liver damage in a dose-dependent manner nonaffecting its potential cytotoxic effect [34]. Hesperidin application 
produced a potent protection against CIS alterations in antioxidant enzymes levels by turning them to normal. But, a low dose of hesperidin was unfruitful to exert any consequential raise in GR and CAT activities [35].

Hesperidin's nephroprotector activity was assessed in male Albino rats after intraperitoneal administration of CIS. CIS nephrotoxicity was demonstrated by elevated levels of blood urea nitrogen, high protein excretion in urine, serum creatinine, and reduced levels of creatinine clearance. Then, hesperidin made a significant protection against cisplatin-induced nephrotoxicity in a dose dependent manner [36].

Kumar et al. studied the protective effect of hesperidin to myocardial injury induced by cyclophosphamide (CP) [37]. CP described an important increased level of malonaldehyde (MDA) and reciprocally decreased levels of SOD, GPx, CAT, GSH and GST in cardiac tissue. Hesperidin group showed elevated levels of all cited enzymes and a diminished level of MDA. Similar results with CP were previously published [38].

In addition, another flavonoid, apigenin (API) acts also synergizing the effect with chemotherapeutic agents such as gemcitabine and 5 -fluorouracil in vivo [39,40]. This fact might indicate that hesperidin and/or hesperetin could act in the same direction. Also, quercetin has the capability to reduce the corneal opacity caused by corneal edema that inhibits mitomycin C (MMC)-induced damage to the corneal endothelial cells [40]. Oral administration of diosmine and quercetin was studied on hematological and hepatic toxicity of a single dose of $\mathrm{CP}$ or vinblastine on rats' female albinos' Wistar. In the group of rats treated only with the $\mathrm{CP}$, since the $1^{\text {st }}$ day, it was observed an increase of lipid peroxide (MDA) more than once (120\%) and a decay of hepatic glutathione and enclosing the group receiving the vinblastine (until more than two times of reduction - 120\%). Also, a severe leucopenia and thrombopenia were noted between the $3^{\text {rd }}$ and the $14^{\text {th }}$ days at rats treated by the chemotherapeutic agents alone (CP and vinblastine) [40].

The association of flavonoids with drugs has efficiently reduced the effect of drugs' toxicity. Actually, the aplasic observed with the vinblastine, as well as the leucopenia and thrombopenia of the $\mathrm{CP}$ were totally corrected. Similarly, the authors observed a restoration of rates of peroxide and GSH. They suggested that flavonoids seem to act by activation of the turnover of the GSH and enzymes stimulating particularly glutathione-S-transferases letting the sequestration of the reactive metabolites of the drugs under studying [41].

Choi et al. demonstrated that the pre-treatment with flavonoids, such as quercetin, acting on male SD rats increased the bioavailability of paclitaxel or its water-soluble pro-drug [42]. Moreover, the coadministration with flavones produced a significant increase of the bioavailability of this drug and they suggest that this probably occurred through the inhibition of P-gp or CYP3A [43]. Similar results were demonstrated by altering the pharmacokinetics of paclitaxel by a concomitant use of morin (flavonoid) in rats [44].

A study with apigenin was projected to investigate whether combined therapy with apigenin and gemcitabine enhanced antitumor activity in pancreatic cancer. In studies in vitro, the combined treatment significantly suppressed cell growth and apoptosis through the down-regulation of NF- $\mathrm{KB}$ activity with inhibition of Akt activation in pancreatic cancer cell lines, such as MiaPaca-2 and AsPC-1. In the case of in vivo treatment, the combined therapy augmented tumor growth suppressed through the down-regulation of NF- $\kappa \mathrm{B}$ activity with the inhibition of Akt in tumor tissue. The association of apigenin and gemcitabine increased anti-tumor efficacy through Akt and NF- $\mathrm{kB}$ activity inhibition and apoptosis induction [45].

In a pre-treatment with hesperetin on rats with DMBA-induced mammary gland tumors, significantly diminished the tumor burden and the over-expression of the proliferating cell nuclear antigen (PCNA), even as, recovering the decreased Bcl-2 and increased Bax expression. On the contrary, in the liver of mice previously treated with DMBA, the hesperetin application prevented DNA fragmentation, cleaved caspase- 3 , caspase- 9 and PARP, and decreased Bax expression [46]. This study indicates that hesperetin may act as a pro-apoptotic or anti-apoptotic agent depending on the situation [46]. Daily administration of hesperetin for fifteen weeks suppressed rat colon carcinogenesis during and hereafter DMH initiation [27].

Novel flavonoid - Oncamex demonstrated a strong antitumor effect in breast cancer cell line through the induction of apoptosis and cytotoxicity. In the MCF-7 cells treatment with Oncamex were tracked after $24 \mathrm{~h}$ and $72 \mathrm{~h}$ and showed substantial changes in depletion of cell density and division, nuclear morphology and arising of apoptotic cells. After $72 \mathrm{~h}$ of treatment, abundant apoptotic, phagocytized and dead cells were present. Further investigation of Oncamex's mechanism demonstrated that treatment of MCF-7, MDAMB-231, BT-549 and HBL-100 cells with micromolar concentrations for $8 \mathrm{~h}$ induced dose-dependent, inversely correlated changes with cell viability and cytotoxicity, along with caspase-3/-7 activation, consistent with apoptosis [47].

\section{Perspectives of Flavonoids with Emphases on Hesperidin and Hesperetin from Orange Peels or Juice on Bladder Cancer}

The chemopreventive effects of diosmine and hesperidin, on $\mathrm{N}$-butyl- $\mathrm{N}$-(4-hydroxybutyl) nitrosamine (OHBBN)-induced urinarybladder carcinogenesis in male ICR mice were verified. The applications of the both compounds, isolated or in combination, in the treatment caused an important reduction in the frequency of bladder carcinoma and pre-neoplasia. Orally administration of diosmin and hesperidin significantly decreased the silver-stained-nucleolar-organizer-regionassociated proteins (AgNOR) count and the 5-bromodeoxyuridine (BUdR)-labeling index of several bladder lesions. These observations suggest that diosmin and hesperidin, individually and in association, are effective in suppressing chemical carcinogenesis of the bladder, and that such suppression might be partly related to inhibition of cell proliferation [48]

Many different meta-analyses have discussed an inverse association between citrus fruits intake and the risk of several types of cancers, and among them bladder cancers [49-52]. This is explainable since the most abundant Citrus flavonoid is hesperidin [53]. The orange hesperidin content is $28-42 \mathrm{mg} / \mathrm{g}$ of dry peel and $12-44 \mathrm{mg} / \mathrm{g}$ of waste from orange juice [54]. It is known that the beneficial effects of flavonoids are due to their anti-oxidant properties, which are a key role in fighting several degenerative diseases. Meanwhile, there is recent enhancing evidence linking the pharmacological activity of Citrus flavonoids to their capability to inhibit the activity of intracellular signaling molecules, such as kinases, phosphodiesterases, topoisomerases, and other different regulatory enzymes $[55,56]$.

Table 1 lists recent studies of bioflavonoids and their antiproliferative and anti-cancer activity in bladder cancer treatment. Bioflavonoids mentioned in the table have very similar structure to hesperidin and hesperetin and belong to other flavonoid subclasses. 
Citation: Stanisic D, Costa AF, Fávaro WJ, Tasic L, Seabra AB, et al. (2018) Anticancer Activities of Hesperidin and Hesperetin In vivo and their Potentiality against Bladder Cancer. J Nanomed Nanotechnol 9: 515. doi: 10.4172/2157-7439.1000515

\begin{tabular}{|c|c|c|c|}
\hline Source & Flavonoid & IC $_{50}$ & Ref \\
\hline Cranberry flavonoids & $\begin{array}{c}\text { Quercetin 3-O-glucoside, } \\
\text { 3'-O-methylquercetin } \\
\text { (isorhamnetin), quercetin, and } \\
\text { myrcetin }\end{array}$ & $8-92 \mu \mathrm{M}$ & {$[57]$} \\
\hline Aladdin chemistry Co. Ltd & Quercetin & $40-70 \mu \mathrm{M}$ & {$[58]$} \\
\hline Scutellariae radix & $\begin{array}{c}\text { Baicalin, baicalein and } \\
\text { wogonin }\end{array}$ & $3.4-100 \mu \mathrm{M}$ & {$[59]$} \\
\hline Gosun Biotecchnology Co. & Myricetin & $20-100 \mu \mathrm{M}$ & {$[60]$} \\
\hline Sigma Aldrich & Fisetin & $60-100 \mu \mathrm{M}$ & {$[61]$} \\
\hline Kava Extract & Flavokawain & $25 \mu \mathrm{M}$ & {$[62]$} \\
\hline Grape seed & Proanthocyanidin & $25-50 \mu \mathrm{M}$ & {$[63]$} \\
\hline
\end{tabular}

Table 1: Natural bioflavonoids as a potential bladder cancer inhibitors and prodrugs.

\section{Conclusions and Final Remarks}

Since hesperidin acts directly in the bladder cancer, as stated and discussed in the previous sections, together with the strong indications that extracts of orange peels and orange juice may also show this anticancer activity, tackle the necessity of a detailed study on hesperidin or hesperetin benefits in bladder cancer treatments. One of the strategies would be to investigate hesperidin or hesperetin as coadjuvant of the chemotherapeutics regiment in bladder cancer (e.g., DOX, CIS, MMC, etc.). Perspectives of this research might be great, if we joint our expertise in bladder cancer on animals [57-71] and easiness of obtaining hesperidin and hesperetin in our laboratories. At last bit not at least, this mini-review shows the importance of introducing new, nontoxic and economically feasible natural products for the cancer treatment.

\section{Acknowledgement}

Supports by FAPESP, INOMAT (MCTI/CNPq), NanoBioss (MCTI/CNPq), and Citrosuco Company are kindly acknowledged.

\section{References}

1. Justesen U, Knuthsen P, Leth T (1998) Quantitative analysis of flavonols, flavones, and flavanones in fruits, vegetables and beverages by highperformance liquid chromatography with photo-diode array and mass spectrometric detection. J Chromatogr A 799: 101-110.

2. Garg A, Garg S, Zaneveld LJ, Singla AK (2001) Chemistry and pharmacology of the Citrus bioflavonoid hesperidin. Phytother Res 15: 655-669.

3. Chen M, Gu H, Ye Y, Lin B, Sun L, et al. (2010) Protective effects of hesperidin against oxidative stress of tert-butyl hydroperoxide in human hepatocytes. Food Chem Toxicol 48: 2980-2987.

4. Tanaka T, Tanaka M, Tanaka T Kuno (2012) Cancer chemoprevention by citrus pulp and juices containing high amounts of $\beta$-cryptoxanthin and hesperidin. BioMedical Research International 2012: 516981.

5. Kheradmanda E, Moghaddama AH, Zareb M (2018) Neuroprotective effect of hesperetin and nano-hesperetin on recognition memory impairment and the elevated oxygen stress in rat model of Alzheimer's disease. Biomed Pharmacother 97: 1096-1101.

6. Stanisic D, Costa AF, Cruz G, Durán N, Tasic L (2018) Applications of hesperidin as an anticancer pro-drug: Phytotherapy or an alternative chemotherapy. In: Studies in Natural Products Chemistry Elsevier 6: 18-52.

7. Arafa HMM, Aly HAA, Abd-Ellah MF, El-Refaey HM (2009) Hesperidin attenuates benzo[a] pyrene-induced testicular toxicity in rats via regulation of oxidant/antioxidant balance. Toxicol Ind Health 25: 417-427.

8. Saiprasad G, Chitra P, Manikandan R, Sudhandiran G (2013) Hesperidin alleviates oxidative stress and downregulates the expressions of proliferative and inflammatory markers in azoxymethane-induced experimental colon carcinogenesis in mice. Inflammation Research 62: 425-440.

9. Suzui M, Okuno M, Tanaka T, Nakagama H, Moriwaki H (2002) Enhanced colon carcinogenesis induced by azoxymethane in min mice occurs via a mechanism independent of $\beta$-catenin mutation. Cancer Lett 183: 31-41.
10. Devi KP, Rajavel T, Nabavi SF, Setzer WN, Ahmadi A, et al. (2015) Hesperidin A promising anticancer agent from nature. Ind Crops Prod 76: 582-589.

11. Saiprasad G, Chitra P, Manikandan R, Sudhandiran G (2014) Hesperidin induces apoptosis and triggers autophagic markers through inhibition of Aurora-A mediated phosphoinositide-3-kinase/Akt/mammalian target of rapamycin and glycogen synthase kinase-3 beta signaling cascades in experimental colon carcinogenesis. Eur J Cancer 50: 2489-2507.

12. Zhu Y, Zhu M, Lance $P$ (2012) iNOS signaling interacts with COX-2 pathway in colonic fibroblasts. Exp Cell Res 318: 2116-2127.

13. Zarubin T, Han J (2005) Activation and signaling of the p38 MAP kinase pathway. Cell Res 15: 11-18.

14. Parhiz H, Roohbakhsh A, Soltani F, Rezaee R, Iranshahi M (2015) Antioxidant and anti-inflammatory properties of the citrus flavonoids' hesperidin and hesperetin: an updated review of their molecular mechanisms and experimental models. Phytother Res 29: 323-331.

15. Bentli R, Ciftci O, Cetin A, Unlu M, Basak N, et al. (2013) Oral administration of hesperidin, a citrus flavonone, in rats counteracts the oxidative stress, theinflammatory cytokine production, and the hepatotoxicity induced by the ngestion of 2,3,7,8-tetrachlorodibenzo-p-dioxin (TCDD). Eur Cytokine Netw 24 91-96.

16. Tanaka T, Makita H, Kawabata K, Mori H, Kakumoto M, et al. (1997) Chemoprevention of azoxymethane-induced rat colon carcinogenesis by the naturally occurring flavonoids, diosmin and hesperidin. Carcinogenesis 18 : 957-965.

17. Sakata K, Hirose Y, Qiao Z, Tanaka T, Mori H (2003) Inhibition of inducible isoforms of cyclooxygenase and nitric oxide synthase by flavonoid hesperidin in mouse macrophage cell line. Cancer Lett 199: 139-145.

18. Lentini A, Forni C Provenzano B, Beninati S (2007) Enhancement of transglutaminase activity and polyamine depletion in B16-F10 melanoma cells by flavonoids naringenin and hesperitin correlate to reduction of the in vivo metastatic potential. Amino Acids 32: 95-100.

19. Zhang J, Song J, Wu D, Wang J, Dong W (2015a) Hesperetin induces the apoptosis of hepatocellular carcinoma cells via mitochondrial pathway mediated by the increased intracellular reactive oxygen species, ATP and calcium. Med Oncol 32: 101

20. Zhang J, Wu D, Song J, Wang J, Yi J, et al. (2015b) Hesperetin induce the apoptosis of gastric cancer cells via activating mitochondrial pathway by increasing reactive oxygen species. Dig Dis Sci 60: 2985-2995.

21. Li S, Lin YC, Ho CT, Lin PY, Suzawa M, et al. (2014) Formulated extract from multiple citrus peels impairs dendritic cell functions and attenuates allergic contact hypersensitivity. Int Immunopharmacol 20: 12-23.

22. Lai CS, Li S, Liu CB, Miyauchi Y, Suzawa M, et al. (2013) Effective suppression of azoxymethane-induced aberrant crypt foci formation in mice with citrus peel flavonoids. Mol Nutr Food Res 57: 551-555.

23. Zhang XH, Zhang NN, Meng XB, Zhang Y, Qian Y, et al. (2017) Hesperetin inhibits the proliferation of cerebrally implanted $\mathrm{C} 6$ glioma and involves suppression of HIF-1a/VEGF pathway in rats. Biomed Res 28: 1205-1211.

24. Krishnan G, Subramaniyan J, Subramani PC, Muralidharan B, Thiruvengadam D (2017) Hesperetin conjugated PEGylated gold nanoparticles exploring the potential role in anti-inflammation and anti-proliferation during diethylnitrosamine-induced hepatocarcinogenesis in rats. As J Parma Sci 12 $442-455$.

25. Aranganathan S, Nalini N (2013) Antiproliferative efficacy of hesperetin (Citrus flavanoid) in 1,2-dimethylhydrazineinduced colon cancer. Phytother Res 27 999-1005.

26. Ye L, Chan FL, Chen SA, Leung LK (2012) The Citrus flavonone hesperetin inhibits growth of aromatase-expressing MCF-7 tumor in ovariectomized athymic mice. J Nutr Biochemistry 23: 1230-1237.

27. Aranganathan S, Selvam JP, Sangeetha N, Nalini N (2009a) Modulatory efficacy of hesperetin (Citrus flavanone) on xenobiotic-metabolizing enzymes during 1,2-dimethylhydrazine-induced colon carcinogenesis. Chem Biol Interact 180: 254-261.

28. Rossi M, Edefonti V, Parpinel M, Lagiou P, Franchi M, et al. (2013) Proanthocyanidins and other flavonoids in relation to endometrial cancer risk: a case-control study in Italy. Br J Cancer 109: 1914-1920. 
Citation: Stanisic D, Costa AF, Fávaro WJ, Tasic L, Seabra AB, et al. (2018) Anticancer Activities of Hesperidin and Hesperetin In vivo and their Potentiality against Bladder Cancer. J Nanomed Nanotechnol 9: 515. doi: 10.4172/2157-7439.1000515

Page 6 of 6

29. Massari F, Santoni M, Ciccarese C, Brunelli M, Conti A, et al. (2015) Emerging concepts on drug resistance in bladder cancer: Implications for future strategies. Crit Rev Oncol Hematol 96: 81-90

30. Jin X, Zhang P, Luo L, Cheng H, Li Y, et al. (2016) Efficient intravesical therapy of bladder cancer with cationic doxorubicin nanoassemblies. Int $J$ Nanomedicine 11: 4535-4544

31. Sherif A, Winerdal M, Winqvist $O$ (2018) Immune responses to neoadjuvant chemotherapy in muscle invasive bladder cancer. Bladder Cancer 4: 1-7.

32. Abdel-Raheem IT, Abdel-Ghany AA (2009) Hesperidin alleviates doxorubicininduced cardiotoxicity in rats. J Egypt Natl Canc Inst 21: 175-184.

33. Ahmadi A, Shadboorestan A (2016) Oxidative stress and cancer; the role of hesperidin, a citrus natural bioflavonoid, as a cancer chemoprotective agent Nutr Cancer 68: 29-39.

34. Omar HA, Mohamed WR, Arafa EA, Shehata BA, El Sherbiny GA, et al. (2016) Hesperidin alleviates cisplatin-induced hepatotoxicity in rats without inhibiting its antitumor activity. Pharmacol Rep 68: 349-356.

35. Sahu BD, Kuncha M, Sindhura GJ, Sistla R (2013) Hesperidin attenuates cisplatin-induced acute renal injury by decreasing oxidative stress, inflammation and DNA damage. Phytomedicine 20: 453-460.

36. Sreedevi A, Spandana U, Bharathi K (2012) Effect of hesperidin isolated from orange peels on Cisplatin-induced nephrotoxicity. Int J Pharma Phytochem Res 4: 49-53.

37. Kumar S, Dhankhar N, Kar V, Shrivastava M, Shrivastava S (2011) Myocardia Injury provoked by cyclophosphamide, protective aspect of hesperidin in rats. Int J PharmBiomed Sci 2: 1288-1296.

38. Ahmadi A, Hosseinimehr SJ, Naghshvar F, Hajir E, Ghahremani M (2008) Chemoprotective effects of hesperidin against genotoxicity induced by cyclophosphamide in mice bone marrow cells. Arch Pharm Res 31: 794-797.

39. Hu XY, Liang JY, Guo XJ, Liu L, Guo YB (2015) 5-Fluorouracil combined with apigenin enhances anticancer activity through mitochondrial membrane potential $\left(\Delta \Psi_{\mathrm{m}}\right)$-mediated apoptosis in hepatocellular carcinoma. Clinical and Experimental Pharmacology and Physiology 42: 146-153.

40. Lee YJ (2014) The effect of quercetin in corneal opacity induced by mitomycin-C App Microscopy 44: 88-95.

41. Lahouel M, Boulkour S, Segueni N, Fillaste JP (2004) The flavonoids effect against vinblastine, cyclophosphamide and paracetamol toxicity by inhibition of lipid-peroxidation and increasing liver glutathione concentration. Pathol Biol (Paris) 52: 314-322.

42. Choi JS, Jo BW, Kim YC (2004a) Enhanced paclitaxel bioavailability after ora administration of paclitaxel or prodrug to rats pretreated with quercetin. Euro Eur J Pharm Biopharm 57: 313-318.

43. Choi JS, Choi HK, Shin SC (2004b) Enhanced bioavailability of paclitaxel after oral co-administration with flavone in rats. Int J Pharm 275: 165-170.

44. Choi BC, Choi JS, Han HK (2006) Altered pharmacokinetics of paclitaxel by the concomitant use of morin in rats. Int J Pharm 323: 81-85.

45. Lee SH, Ryu JK, Lee KY, Woo SM, Park JK, et al. (2008) Enhanced anti-tumor effect of combination therapy with gemcitabine and apigenin in pancreatic cancer. Cancer Letters 259: 39-49.

46. Choi EJ, Kim GH (2011) Anti-/pro-apoptotic effects of hesperetin against 7 12-dimetylbenz(a)anthracene induced alteration in animals. Oncol Rep 25 : 545-550.

47. Martínez-Pérez C, Ward C, Turnbull AK, Mullen P, Cook G, et al. (2016) Antitumour activity of the novel flavonoid Oncamex in preclinical breast cancer models. Br J Cancer 114: 905-916.

48. Yang M, Tanaka T, Hirose Y, Deguchi T, Mori H, et al. (1997) Chemopreventive effects of diosmin and hesperidin on N-butyl-N-(4-hydroxybutyl) nitrosamineinduced urinary-bladder carcinogenesis in male ICR mice. Int J Cancer 73 719-724.

49. Xu C, Zeng XT, Liu TZ, Zhang C, Yang ZH, et al. (2015) Fruits and vegetables intake and risk of bladder cancer: A prisma-compliant systematic review and dose-response meta-analysis of prospective cohort studies. Medicine 94: e759.

50. Petrick JL, Steck SE, Bradshaw PT, Trivers KF, Abrahamson PE, et al. (2015) Dietary intake of flavonoids and esophageal and gastric cancer: incidence and survival in the United States of America (USA). Br J Cancer 112: 1291-1300.
51. Yao B, Yan $Y$, Ye X, Fang $H, X u H$, et al. (2014) Intake of fruit and vegetables and risk of bladder cancer: A dose-response meta-analysis of observational studies. Cancer Causes Control 25, 1645-1658.

52. Liang S, Lv G, Chen W, Jiang J, Wang J (2014) Citrus fruit intake and bladder cancer risk: A meta-analysis of observational studies. Int J Food Sci Nutr 65 893-898.

53. Cirmi S, Ferlazzo N, Lombardo GE, Maugeri A, Calapai G, et al. (2016) Chemopreventive agents and inhibitors of cancer hallmarks: May citrus offer new perspectives? Nutrients 8: E698.

54. Pereira RMS, López BGC, Diniz SN, Antunes AA, Garcia DM, et al. (2017) Quantification of flavonoids in Brazilian orange peels and industrial orange juice processing wastes. Agricultural Science 8: 631-644.

55. Rawson NE, Ho CT, Li S (2014) Efficacious anti-cancer property of flavonoids from citrus peels. Food Science and Human Wellness 3: 104-109.

56. Benavente-Garcia O, Castillo J (2008) Update on uses and properties of citrus flavonoids: New findings in anticancer, cardiovascular, and anti-inflammatory activity. J Agric Food Chem 56: 6185-6205.

57. Prasain JK, Rajbhandari R, Keeton AB, Piazza GA, Barnes S (2016) Metabolism and growth inhibitory activity of cranberry derived flavonoids in bladder cancer cells. Food Funct 7: 4012-4019.

58. Su Q, Peng M, Xhang Y, Xu W, Darko KW, et al. (2016) Quercetin induces bladder cancer cells apoptosis by activation of AMPK signaling pathway. Am J Cancer Res 6: 498-508.

59. Ikemoto S, Sugimura K, Yoshida N, Yasumoto R, Wada S, et al. (2000) Antitumor effects of Scutellariae radix and its components baicalein, baicalin and wogonin on bladder cancer cell lines. Urology 55: 951-995.

60. Sun F, Zheng XY, Ye J, Wu TT, Wang Jl, et al. (2012) Potential Anticancer Activity of Myricetin in Human T24 Bladder Cancer Cells Both In Vitro and In Vivo. Nutr Cancer 64: 599-606

61. Li J, Cheng Y, Qu W, Sun Y, Wang Z, et al. (2010) Fisetin, a Dietary Flavonoid, Induces Cell Cycle Arrest and Apoptosis through Activation of p53 and Inhibition of NF-Kappa B Pathways in Bladder Cancer Cells. Basic Clin Pharmacol Toxicol 108: 84-93.

62. Zi X1, Simoneau AR (2005) Flavokawain A, a novel chalcone from kava extract, induces apoptosis in bladder cancer cells by involvement of Bax proteindependent and mitochondria-dependent apoptotic pathway and suppresses tumor growth in mice. Cancer Res 65: 3479-3486.

63. Fishman Al, Johnson B, Alexander B, Won J, Choudhury M, et al. (2012) Additively Enhanced Antiproliferative Effect of Interferon Combined with Proanthocyanidin on Bladder Cancer Cells. J Cancer 3: 107-112.

64. Durán N, Fávaro WJ (2018) Nanopharmaceuticals and their applications in bladder cancer therapy: A mini review. J the Brazilian Chemical Society 29 973-981.

65. Seabra AB, Durán N (2018) Nitric oxide donors for prostate and bladder cancers: Current state and challenges. Eur J Pharmacol 826: 158-168.

66. Da Silva RC, de Souza JG, Seabra AB, Durán N, Fávaro WJ (2018) Advances and perspectives in urinary bladder cancer nanotherapy. Eur Medical J 6: 57-61.

67. Dias LP, Luzo ACM, Volpe BB, Galdames SEM, de Jesus MB, et al. (2018) Effects of intravesical therapy with platelet-rich plasma (PRP) and Bacillus CalmetteGuérin (BCG) in non-muscle invasive bladder cancer. Tissue Cell 52: 17-27.

68. Garcia PV, Seiva FRF, Carniato AP, de Mello JW, Durán N, et al. (2016) Invasive bladder cancer: Mechanism of action of P-MAPA biological response modifier increased toll-like receptors and p53 Levels regulate apoptosis and angiogenesis in non-muscle invasive bladder cancer. BMC Cancer 16: 422.

69. Garcia PV, Apolinário LM, Böckelmann PK, Nunes IS, Durán N, et al. (2015) Alterations in ubiquitin ligase Siah-2 and its corepressor N-CoR after P-MAPA immunotherapy and anti-androgen therapy: New therapeutic opportunities fo non-muscle invasive bladder cancer. Int J Clin Exp Pathol 8: 4427-4443.

70. Fávaro WJ, Nunes OS, Seiva FRF, Nunes IS, Woolhiser LK, et al. (2012) Effects of P-MAPA immunomodulator on Toll-like receptors and p53: Potential terapeutic srategies for infectious diseases and câncer. Infect Agent Cance 7: 14

71. Favaro WJ, Billis A Nunes IS, Durán N (2012) New immunotherapy for nonmuscle invasive bladder cancer (NMIBC): effects of immunomodulator P-MAPA. J Urology 187: E231-232. 